\title{
Scalable TFETI domain decomposition based contact algorithm
}

\author{
J. Dobiášs ${ }^{1}$, S. Pták ${ }^{1}$, Z. Dostál ${ }^{2}$, T. Kozubek ${ }^{2}$ \& A. Markopoulos ${ }^{2}$ \\ ${ }^{1}$ Institute of Thermomechanics, Prague, Czech Republic \\ ${ }^{2}$ University of Ostrava, Czech Republic
}

\begin{abstract}
This paper is concerned with a novel algorithm for a solution to contact problems stemming from the TFETI (Total Finite Element Tearing and Interconnecting) domain decomposition method. The TFETI method is based on the idea that the compatibility between non-overlapping sub-domains, into which the original domain is partitioned, is enforced by the Lagrange multipliers. The distinctive feature of the TFETI consists of the fact that the method also enforces the Dirichlet boundary conditions by means of the Lagrange multipliers. The TFETI based technique converts the original contact problem to the quadratic programming one with the equalities and simple bound constraints. Moreover, it also results in more efficient preconditioning by an enriched natural coarse grid defined by a priory known kernels of the stiffness matrices. Our new algorithm exhibits both parallel and numerical scalabilities so that it enables us to effectively solve steady-state problems of deformable bodies undergoing contact, geometric and material nonlinear effects. In this paper we propose an algorithm with nested iteration strategy, where its inner part consists of a new version of our previously developed MPRGP and SMALBE algorithms and the outer loop iterates on the geometric and material non-linearities. Numerical experiments include solutions to steady-state problems with non-linear effects and their results document that the proposed algorithms are robust, highly accurate and exhibit both parallel and numerical scalabilities.

Keywords: contact non-linearity, geometric non-linearity, material non-linearity, domain decomposition, scalability.
\end{abstract}




\section{Introduction}

Dostál et al. analysed in [1] problem of frictionless contact problem between solid bodies, while they considered both geometrically and materially linear cases. Therein they suggested a new in a sense optimal version of their own previously developed algorithm based on TFETI (Total Finite Element Tearing and Interconnecting) domain decomposition method. The goal of this paper is to apply this new algorithm to the contact problems accompanied by both geometric and material non-linear phenomena, and to show that it can yield, even under these conditions, good results.

The FETI domain decomposition method was introduced by Farhat and Roux [2] as a parallel finite element solver for the self-adjoint elliptic partial differential equations. Its key idea is a decomposition of the spatial domain into nonoverlapping sub-domains that are 'glued' by Lagrange multipliers, so that, after eliminating the primal variables, or displacements, the original problem is reduced to a small, relatively well conditioned, typically equality constrained quadratic programming problem that is to be solved iteratively. Later Farhat et al. modified the basic FETI algorithm so that they were able to prove its numerical scalability, i.e. asymptotically linear complexity.

The partition of the original domain into sub-domains usually generates some 'floating' sub-domains with not enough prescribed displacements, so that their stiffness matrices are singular in steady-state cases and implementation of FETI then includes the computation of their kernels. However, stable evaluation of the bases of the kernels, though theoretically clear in exact arithmetic context, is tricky in the presence of the round-off errors. To overcome this difficulty, Dostál et al. [4] suggested enforcement of all the Dirichlet boundary conditions by the Lagrange multipliers so that all the sub-domains were treated as totally unconstrained. This version of FETI is referred to as the Total FETI (TFETI). Since the kernels of stiffness matrices of all the sub-domains are the same and known beforehand, this approach removed the problems with identification of these kernels.

Even though the FETI class methods were originally developed for numerical solution to linear elliptic partial differential equations, it turned out that they were even more successful for the solution to contact problems. The reason is that any FETI method reduces in effect for free the more general inequalities introducing the conditions of non-penetration of bodies to the bound constraints. The FETI based algorithm for contact problems was proposed by Dostál et al. in [5]. The numerical scalability of this algorithm was demonstrated by numerical experiments.

The organisation of the paper is as follows. Section 2 introduces the TFETI for contact problems. Section 3 is briefly concerned with a new version of SMALBE and MPRGP, which are the algorithms stemming from the TFETI. The concepts of numerical and parallel scalabilities are explained in Section 4. Section 5 outlines the overall computational strategy and we present there an algorithm that enables TFETI to be applied to solution to the contact, geometric and material non-linear 
effects. Section 6 shows results of numerical experiments and Section 7 concludes the paper.

\section{Problem formulation}

For simplicity, let us consider a system of two solid deformable bodies in contact which occupy in reference configuration domain $\Omega=\Omega^{m} \cup \Omega^{s}$, where $m$ denotes master body and $s$ stands for slave body. Assume that their boundaries are subdivided into three disjoint parts $\Gamma_{u}^{i}, \Gamma_{f}^{i}$, and $\Gamma_{c}^{i}, i=m, s$, with prescribed Dirichlet, Neumann, and potential contact conditions, respectively.

The strong version of governing equations of the static equilibrium is as follows

$$
\sigma_{i j}(\mathbf{u})_{, j}=f_{i} \quad \text { in } \Omega,
$$

where $\boldsymbol{\sigma}, \mathbf{u}$, and $\mathbf{f}$ denote stress tensor, displacement vector and loading vector, respectively. The Dirichlet and Neumann boundary conditions are, respectively, as reads

$$
\begin{gathered}
\mathbf{u}=0 \quad \text { on } \quad \Gamma^{u}, \\
\sigma_{i j}\left(\mathbf{u} \cdot \nu_{j}\right) \mathbf{u}=p_{i} \quad \text { on } \quad \Gamma^{f} .
\end{gathered}
$$

There are several conditions characterising the contact. Consider the frictionless contact. The first is the kinematic contact condition which says that solids cannot penetrate each other so that the gap $g$ between them is either zero or positive

$$
g \geq 0 \quad \text { on } \quad \Gamma^{c}
$$

The second one is the mechanical contact condition which postulates that solids cannot pull on each other in the absence of adhesion so that only compressive normal stresses $\sigma_{\nu}$ are allowed

$$
\sigma_{\nu} \leq 0 \quad \text { on } \quad \Gamma^{c}
$$

The complementarity or exclusivity conditions asserts that solids press on each other or they are separated

$$
\sigma_{\nu} \cdot g=0 \quad \text { on } \quad \Gamma^{c}
$$

The variational formulation is to be obtained if we consider the constrained minimisation problem

$$
\mathbf{u} \in \mathcal{K}, \quad \mathcal{J}(u) \leq \mathcal{J}(v) \quad \forall v \in \mathcal{K},
$$

where $\mathcal{K}$ is a non-empty, closed, convex set of all feasible displacements. The quadratic functional

$$
\mathcal{J}(\mathbf{v})=\frac{1}{2} a(\mathbf{v}, \mathbf{v})-\ell(\mathbf{v})
$$


is generated by a self-adjoint, positive definite bilinear form

$$
a(\mathbf{v}, \mathbf{w})=\sum_{i \in\{s, m\}} \int_{\Omega^{i}} \boldsymbol{\sigma}\left(\mathbf{v}^{i}\right): \boldsymbol{\epsilon}\left(\mathbf{w}^{i}\right) d x
$$

and accounts for internal forces. The linear functional

$$
\ell(\mathbf{v})=\sum_{i \in\{s, m\}} \int_{\Omega^{i}} \mathbf{f} \cdot \mathbf{v} d x+\sum_{i \in\{s, m\}} \int_{\Gamma^{i}}^{f} \mathbf{p} \cdot \mathbf{v} d x
$$

accounts for the volumetric forces and tractions on the Neumann boundary.

To apply the TFETI domain decomposition, we tear each body from the part of the boundary with the prescribed Dirichlet boundary conditions, decompose each body into sub-domains and introduce new connecting conditions on the fictitious intersubdomain boundaries and on boundaries with imposed Dirichlet conditions. The connecting conditions requires continuity of the displacements and of their normal derivatives across the intersubdomain boundaries.

Applying the standard finite element procedures while in addition considering TFETI method, we can express the governing equations of a contact problem in discretised form as follows

$$
\begin{gathered}
\mathbf{K u}=\mathbf{f}-\mathbf{B}_{I}^{\top} \boldsymbol{\lambda}_{I}-\mathbf{B}_{E}^{\top} \boldsymbol{\lambda}_{E}, \\
\mathbf{B}_{I} \mathbf{u} \leq \mathbf{c}_{I}, \\
\mathbf{B}_{E} \mathbf{u}=\mathbf{c}_{E},
\end{gathered}
$$

where $\mathbf{K}$ denotes a stiffness matrix with sparse positive semidefinite diagonal blocks corresponding to individual sub-domains. In accordance with the TFETI method, the kernels of all the sub-domains are the same and known beforehand. The matrix $\mathbf{B}_{I}$ and the vector $\mathbf{c}_{I}$ introduce the linearised non-interpenetration conditions. Similarly the matrix $\mathbf{B}_{E}$ and the vector $\mathbf{c}_{E}$ enforce the prescribed displacements along the part of the boundary with the Dirichlet conditions. $\boldsymbol{\lambda}_{I}$ and $\lambda_{E}$ stand for the components of the vector of Lagrangean multipliers, while we shall use

$$
\boldsymbol{\lambda}=\left[\begin{array}{c}
\boldsymbol{\lambda}_{I} \\
\boldsymbol{\lambda}_{E}
\end{array}\right], \quad \mathbf{B}=\left[\begin{array}{c}
\mathbf{B}_{I} \\
\mathbf{B}_{E}
\end{array}\right] \quad \text { and } \quad \mathbf{c}=\left[\begin{array}{c}
\mathbf{c}_{I} \\
\mathbf{c}_{E}
\end{array}\right] .
$$

Eqn. (11a) has a solution iff $\mathbf{f}-\mathbf{B}^{\top} \boldsymbol{\lambda}$ belongs to the range of $\mathbf{K}$ and therefore the following relationship holds

$$
\mathbf{R}^{\top}\left(\mathbf{f}-\mathbf{B}^{\top} \boldsymbol{\lambda}\right)=\mathbf{0}
$$

where $\mathbf{R}$ denotes the full rank matrix with columns spanning the kernel of $\mathbf{K}$. Since all the sub-domains are totally unconstrained, the kernels of the sub-domains are known beforehand and are to be assembled directly. 
It is necessary to eliminate the primal variable $\mathbf{u}$ from eqn. (11a). It can easily be verified that if $\mathbf{u}$ is a solution to eqn. (11a), then there exists such a vector $\boldsymbol{\alpha}$ that

$$
\mathbf{u}=\mathbf{K}^{\dagger}\left(\mathbf{f}-\mathbf{B}^{\top} \boldsymbol{\lambda}\right)+\mathbf{R} \boldsymbol{\alpha},
$$

where $\mathbf{K}^{\dagger}$ is any symmetric positive definite matrix satisfying the first MoorePenrose condition $\mathbf{K K}^{\dagger} \mathbf{K}=\mathbf{K}$, or it is the $\{1\}$-inverse.

Substituting eqn. (13) into eqn. (11a), we get the following minimisation problem

$\min \frac{1}{2} \boldsymbol{\lambda}^{\top} \mathbf{B} \mathbf{K}^{\dagger} \mathbf{B}^{\top} \boldsymbol{\lambda}-\boldsymbol{\lambda}^{\top} \mathbf{B} \mathbf{K}^{\dagger} \mathbf{f} \quad$ s. t. $\quad \boldsymbol{\lambda}_{I} \geq \mathbf{0} \quad$ and $\quad \mathbf{R}^{\top}\left(\mathbf{f}-\mathbf{B}^{\top} \boldsymbol{\lambda}\right)=\mathbf{0}$.

Let us now introduce the standard FETI notations

$$
\mathbf{F}=\mathbf{B K}^{\dagger} \mathbf{B}^{\top}, \quad \mathbf{G}=\mathbf{R}^{\top} \mathbf{B}^{\top}, \quad \mathbf{e}=\mathbf{R}^{\top} \mathbf{f}, \quad \mathbf{d}=\mathbf{B} \mathbf{K}^{\dagger} \mathbf{f} .
$$

After some algebraic manipulation, we obtain the following formulation

$$
\min \frac{1}{2} \boldsymbol{\lambda}^{\top} \mathbf{P F P} \boldsymbol{\lambda}-\boldsymbol{\lambda}^{\top} \mathbf{P d} \quad \text { s. t. } \quad \boldsymbol{\lambda}_{I} \geq \mathbf{0} \quad \text { and } \quad \mathbf{G} \boldsymbol{\lambda}=\mathbf{0},
$$

where

$$
\mathbf{P}=\mathbf{I}-\mathbf{Q} \quad \text { and } \quad \mathbf{Q}=\mathbf{G}^{\top}\left(\mathbf{G G}^{\top}\right)^{-\mathbf{1}} \mathbf{G}
$$

stand for the orthogonal projectors on the kernel of $\mathbf{G}$ and the range of $\mathbf{G}^{\top}$, respectively. The point of the last step is to introduce preconditioning by the natural coarse grid projector.

The problem (16) is to be solved efficiently by the algorithms presented in the following section.

\section{MPRGP and SMALBE algorithms}

We have shown that application of the TFETI methodology to the contact problems converts the original problem to the quadratic programming problem with bound and equality constraints and well-conditioned regular part of the Hessian matrix. Such problems are to be solved very efficiently by the recently proposed algorithms. A unique and qualitatively new feature of these algorithms is the rate of convergence in the bounds on the regular part of the Hessian, independent of the representation of constraints. See [1] for details.

The problem (16) is first reduced by Semi-Monotonic Augmented Lagrangeans with Bound and Equality constraints (SMALBE) method to the sequence of bound constrained quadratic programming problems. SMALBE accepts inexact solutions of auxiliary bound constrained problems solved approximately in the inner loop until the norm of the projected gradient is proportional to the feasibility error, and updates the regularisation parameter until the value of the Lagrangian increases. These auxiliary problems are to be solved efficiently by the Modified Proportioning with Reduced Gradient Projection (MPRGP) method in the inner 
loop. It was proved by Dostál and Horák [6] that application of FETI or TFETI with natural coarse grid preconditioning to a contact problem can be combined with SMALBE and MPRGP to obtain algorithm with asymptotically linear, i.e. optimal, complexity. A unique feature of MPRGP is the rate of convergence which is independent of the inequality constraints. Moreover, the algorithm enjoys the finite termination property even for dual degenerate problems, so that it does not suffer from oscillations often attributed to this type of algorithms.

Detailed description of these algorithms is beyond the scope of this paper.

\section{Numerical and parallel scalabilities}

The performance of a domain decomposition based iterative method depends on two important properties, namely numerical and parallel scalabilities. Such a method is said to be numerically scalable if the condition number of the problem does not grow or grows weakly with the ratio of the sub-domain size and the mesh size. The parallel scalability represents ability of an algorithm to achieve larger speed-ups for a larger number of processes.

\section{Non-linear algorithm}

The primary interest of this work is the development of effective strategy for fully non-linear problems, where, in addition to the contact interaction, the kinematics of the body system are not confined to small strains, and where the material response is potentially non-linear and inelastic.

The strains can be evaluated by means of the following in general non-linear relationship

$$
\boldsymbol{\epsilon}=\mathbf{B}_{\mathbf{s}}(\mathbf{u}) \cdot \mathbf{u}
$$

where $\mathbf{B}_{s}$ denotes an appropriate matrix relating strains with nodal displacements.

The stresses are computed by solving this constitutive equation

$$
\boldsymbol{\sigma}=\sum_{n e l e m} \int_{0}^{\boldsymbol{\epsilon}} \mathbf{D} \mathrm{d} \boldsymbol{\epsilon}
$$

where D stands for the tangent material matrix. Its particular form depends on applied material model. We sum over nelem elements.

The MPRGP and SMALBE algorithms are directly applicable to solution to the contact problem, which itself is a strongly non-linear phenomenon indeed, but with other conditions linear. Any additional non-linear effect necessitates employment of the nested iteration strategy, where the inner loop is concerned with TFETI based solver, while the outer loop iterates on the material/geometric non-linear effects and contact geometry update so that it might achieve equilibrium.

Consider the state of equilibrium of the system. Employing the modified Newton-Raphson iterative method, the governing equilibrium equations is as 
follows

$$
\mathbf{K}^{(i-1)} \Delta \mathbf{u}^{(i)}=\mathbf{f}_{e x t}-\mathbf{f}_{i n t}^{(i-1)}
$$

$\mathbf{K}, \mathbf{f}_{e x t}$ and $\mathbf{f}_{i n t}$ denote the stiffness matrix, the vector of external nodal loading, and vector of internal nodal forces, respectively. The right superscript $(i)$ stands for the current number of iteration. $\Delta \mathbf{u}^{(i)}$ denotes the displacement increment at each iteration, while the total displacement is updated as follows

$$
\mathbf{u}^{(i)}=\mathbf{u}^{(i-1)}+\Delta \mathbf{u}^{(i)}
$$

Then we can in turn compute the strain tensor, stress tensor and internal forces as reads

$$
\begin{gathered}
\boldsymbol{\epsilon}^{(i)}=\mathbf{B}_{s}\left(\mathbf{u}^{(i)}\right) \mathbf{u}^{(i)}, \\
\boldsymbol{\sigma}^{(i)}=\sum_{\text {nelem }} \int_{0}^{\boldsymbol{\epsilon}^{(i)}} \mathbf{D} \mathrm{d} \boldsymbol{\epsilon}, \\
\mathbf{f}_{\text {int }}^{(i)}=\sum_{\text {nelem }} \int_{\Omega^{(i)}} \mathbf{B}_{\mathbf{s}}^{\top}\left(\mathbf{u}^{(i)}\right) \boldsymbol{\sigma}\left(\boldsymbol{\epsilon}^{(i)}\right) \mathrm{d} \Omega^{(i)} .
\end{gathered}
$$

Equations (20) to (22) describe the problem in terms of the primal variables, i.e. displacements. The stiffness matrix and RHS vector of eqn. (20) have to be transformed in the sense of Section 2 in order that the problem might be computed by MPRGP/SMALBE algorithms for the Lagrangian multipliers. Then we can return to the primary variables, i.e. the displacement increments.

The simplified solution algorithm is shown in the following flowchart.

Initial step: Assemble stiffness matrix $\mathbf{K}$ and $\mathbf{B}_{E}$;

Set $i=0, \quad \mathbf{u}^{0}=0, \quad \mathbf{f}_{\text {int }}^{0}=0$;

Step 1: Evaluate contact conditions $\mathbf{B}_{I}^{(i)}$;

Step 2: Solve contact problem by MPRGP/SMALBE for $\boldsymbol{\lambda} \rightarrow \Delta \mathbf{u}$, $\mathbf{u}^{(i)}=\mathbf{u}^{(i-1)}+\Delta \mathbf{u}$.

Step 3: Compute the strain tensor $\boldsymbol{\epsilon}^{(i)}$ and stress tensor $\boldsymbol{\sigma}^{(i)}$.

Step 4: Integrate the equivalent internal forces $\mathbf{f}_{i n t}^{(i)}$.

Step 5: Assemble the residual load vector $\operatorname{res}^{(i)}=\mathbf{f}_{i n t}^{(i)}-\mathbf{f}_{e x t}$;

check on convergence criteria $\frac{\|\Delta \mathbf{u}\|}{\left\|\mathbf{u}^{(i)}\right\|}<\eta_{1}, \quad \frac{\left\|\mathbf{r e s}^{(i)}\right\|}{\left\|\mathbf{f}_{\text {ext }}\right\|}<\eta_{2} ;$

If fulfilled then STOP, otherwise set $i \leftarrow i+1$ and go to Step 1 .

\section{Numerical experiments}

This section presents results of two sets of numerical experiments with the proposed algorithms. The first one is concerned with analysis of a bolt and nut 


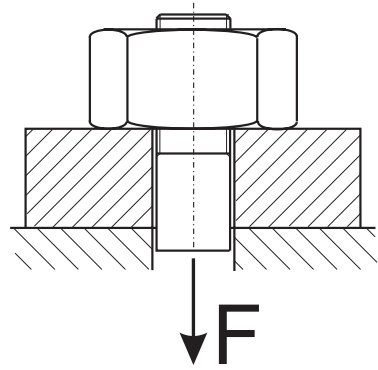

(a)

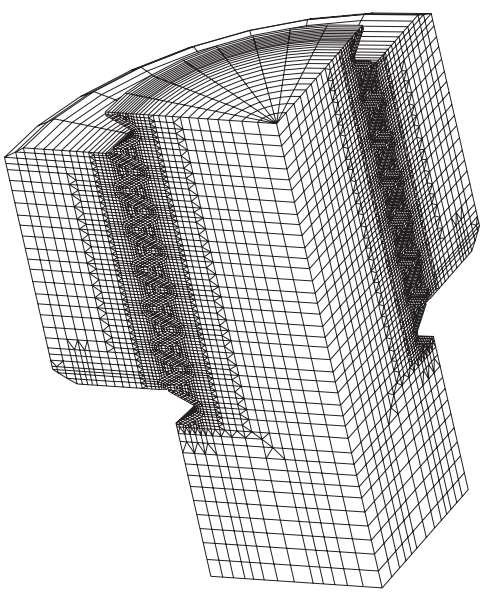

(b)

Figure 1: Bolt \& nut problem; (a) schematic sketch, (b) mesh.

contact problem and the second one shows results concerning the numerical and parallel scalabilities. All the numerical experiments were carried out with our inhouse general purpose finite element package PMD (Package for Machine Design) $[7]$.

\subsection{Bolt and nut contact problem}

Consider a bolt and nut of $30 \mathrm{~mm}$ diameter and the metric thread. The nut rests on a washer and the bolt is loaded by a force $\mathbf{F}$ according to fig. 1(a). The problem is semi-coercive in the sense that we did not prescribe any constraints for the bolt in the direction of its axis. The motion of the bolt is restricted only by the surfaces in contact. Fig. 1(b) shows the finite element mesh, which was strictly created in accordance with the corresponding technical standard, apart from the thread that is not in form of the helix but rings. The problem is modelled with linear penta/hexahedra and we modelled only one quarter because of the symmetry. Numbers of primal and dual variables are 95052 and 1296, respectively, for the case with decomposition into two sub-domains. We consider both the geometric and material non-linear effects. The material properties for linearly-elastic-perfectly-plastic material model are as follows: Young's modulus $E=2.1 \times 10^{11} \mathrm{~Pa}$, Poisson's ratio $\nu=0.3$ and yield stress $\sigma_{Y}=250 \mathrm{MPa}$. We used the von Mises yield criterion.

Fig. 2 shows distribution of von Mises stresses for three levels of loading. The first column depicts results for load $F=3.8 \mathrm{kN}$, the second one for $F=11.3 \mathrm{kN}$, and the third one for $F=22.6 k N$. The upper row represents results with applied elastic material model and the lower one with the plastic model. 

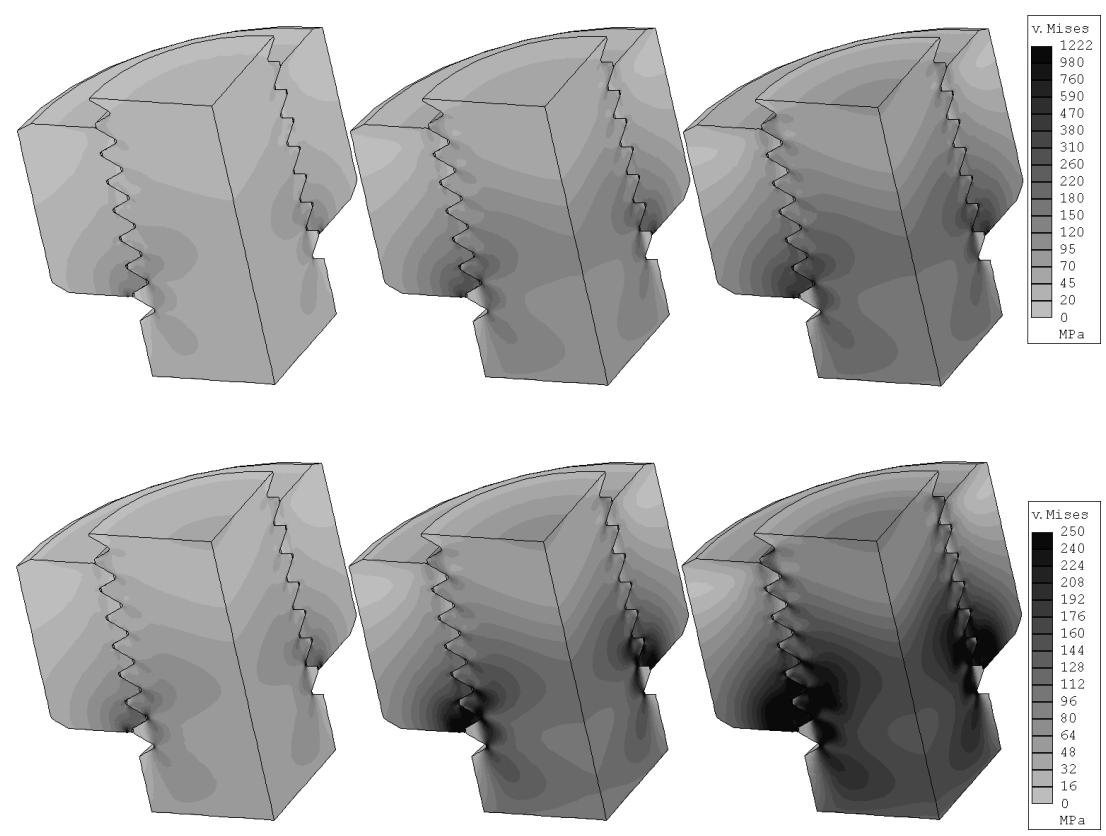

Figure 2: Von Mises stress distribution.

\subsection{Numerical and parallel scalabilities}

Consider contact of two identical elastic cubes of size $a=10 \mathrm{~mm}$ and of the following material properties: Young's modulus $E=2.1 \times 10^{11} \mathrm{~Pa}$ and Poisson's ratio $\nu=0.3$. The computational model is discretised by tri-linear cubical elements and decomposed into cubical sub-domains, while their ratio $\frac{H}{h}=10$. Considering parallel treatment, each sub-domain is assigned one processor.

The upper part of table 1 shows numbers of primal and dual variables for various decompositions. The lowest row demonstrates the numerical scalability of our algorithm. It reads numbers of the Hessian multiplications, which are the most important indicators of the performance of the algorithm and correspond to the numbers of iterations. It is obvious that the number of iterations increases only mildly with refinement of the mesh in the case that the ratio $\frac{H}{h}$ is held constant, which is in good agreement with theory.

It is clear from table 2 that our algorithm exhibits the parallel scalability.

\section{Conclusion}

New version of the algorithms MPRGP and SMALBE stemming from the TFETI domain decomposition method were applied to solution to contact problems accompanied by geometric and material non-linear effects. It was shown that 
Table 1: Numerical scalability.

\begin{tabular}{|l|r|r|r|r|}
\hline Subdomains & 16 & 54 & 128 & 250 \\
\hline Primal variables & 196608 & 663552 & 1572864 & 3072000 \\
\hline Dual variables & 21706 & 81652 & 214699 & 443920 \\
\hline Hessian multiplications & 60 & 63 & 67 & 69 \\
\hline
\end{tabular}

Table 2: Parallel speed-up.

\begin{tabular}{|l|r|r|r|r|r|}
\hline Processors & 2 & 4 & 8 & 16 & 24 \\
\hline Solver & 1.76 & 3.66 & 7.57 & 15.30 & 22.89 \\
\hline Preprocessor & 1.70 & 3.61 & 7.55 & 15.24 & 22.81 \\
\hline Total & 1.73 & 3.63 & 7.56 & 15.27 & 22.85 \\
\hline
\end{tabular}

they yield accurate solution, their converge rate is high and they exhibit both numerical and parallel scalabilities, which is essential for their application to the high performance computers.

\section{Acknowledgements}

The authors would like to acknowledge the support of GA CR through grant number 101/08/0574 and AS CR through grant number AV0Z20760514.

\section{References}

[1] Dostál, Z., Kozubek, T., Vondrák, V., Brzobohatý, T. \& Markopoulos, A., Scalable TFETI algorithm for the solution of contact problems of elasticity. International Journal for Numerical Methods in Engineering, 82(11), pp. 1384-1405, 2010.

[2] Farhat, C. \& Roux, F.X., A method of finite element tearing and interconnecting and its parallel solution algorithm. International Journal for Numerical Methods in Engineering, 32(12), pp. 1205-1227, 1991.

[3] Farhat, C., Mandel, J. \& Roux, F.X., Optimal convergence properties of the FETI domain decomposition method. Computer Methods in Applied Mechanics and Engineering, 115(5), pp. 365-385, 1994.

[4] Dostál, Z., Horák, D. \& Kučera, R., Total FETI - an easier implementable variant of the FETI method for numerical solution of elliptic PDE. 
Communications in Numerical Methods in Engineering, 22(6), pp. 11551162, 2006.

[5] Dostál, Z., Horák, D., Kučera, R., Vondrák, V., Haslinger, J., Dobiáš, J. \& Pták, S., FETI based algorithms for contact problems: scalability, large displacements and 3D Coulomb friction. Computer Methods in Applied Mechanics and Engineering, 194(2-5), pp. 395-409, 2005.

[6] Dostál, Z. \& Horák, D., Theoretically supported scalable FETI for numerical solution of variational inequalities. SIAM Journal of Numerical Analysis, 45(6), pp. 500-513, 2007.

[7] PMD, www.it.cas.cz/manual/pmd 